\title{
CURRENT HIGH ENERGY EMISSION FROM BLACK HOLES
}

\author{
R. D. BLANDFORD \\ 130-33 Caltech \\ Pasadena, CA 91125, USA \\ E-mail: rdb@caltech.edu
}

\begin{abstract}
Two related topics are discussed. 1. Accretion onto black holes at low and high (though not very high) rates is believed to proceed adiabatically (ie nonradiatively). It is argued that the liberated energy is carried off by an outflow, probably involving almost all of the gas that is supplied. Two dimensional, fluid, accretion disks, in which mass, angular momentum and energy are transported to the disk surface, are summarized. It is conjectured that relatively minor changes are needed to describe magnetised disks. By contrast, the disk surface physics is argued to dictate the character of the outflow. 2. Ultrarelativistic jets appear to be produced by active galactic nuclei (AGN), pulsars and $\gamma$-ray bursts (GRB). In all three cases, it is argued that the power is generated electromagnetically by a magnetic rotator, (in a DC not $\mathrm{AC}$ form), and transported in this manner to the emission site. A model of a relativistically expanding electromagnetic shell is described and used to provide a simple model of a GRB in which the $\gamma$-rays are produced by unstable electrical currents flowing along the rotation axis. The shell drives a relativistic blast wave into the surrounding medium with a speed that varies with latitude and whose afterglow emission may exhibit achromatic breaks. Similar processes may be at work in non-relativistic plerions like the Crab Nebula and, possibly, AGN jets. The observational implications of these two classes of model and the prospects for performing instructive, numerical experiments to elucidate them further are briefly outlined.
\end{abstract}

\section{Introduction}

I would like to take literally the title of this meeting and summarize two, related, theories of how black holes (and, to some extent, magnetised neutron stars) behave. The first, which draws upon research carried out with Mitch Begelman, is a particular viewpoint on how accretion proceeds at rates that range from low to high. The second, which is summarised in greater depth elsewher , involves collaboration with Max Lyutikov. It contains some new approaches to describing how electromagnetic Poynting flux is released by a black hole (or indeed any compact spinning, magnetised body) and how it propagates to a remote site from which observable radiation is emitted. I should caution the reader that these are both active and controversial research areas and there are many other points of view that I shall not review. However, I will try to highlight one or two areas where I think that the differences between models are sharply delineated and where I anticipate that progress is imminent. 


\section{Ondol Dynamics}

\subsection{Modes of Accretion}

Accretion $a^{a}$ onto a black hole is approximately scale-free, in the sense that the mode of the accretion is most strongly determined by the ratio of the mass supply rate to the Eddington rate $\left(\dot{M}_{\mathrm{E}}=4 \pi G M / \kappa_{T} c\right)$ and the product of the angular velocity of the hole, $\Omega$, with its mass $M$ [Ferrarese; henceforth, other contributors to these proceedings will be designated using square brackets]. (It is assumed that the gas has sufficient angular momentum to form a disk.) There will be some sensitivity to the mass through the effective temperature. The mass supply rate is usually estimated by the Bondi rate $\dot{M}_{B} \sim \pi r_{B}^{2} \rho_{B} s_{B} \sim \dot{M}_{E} \tau_{B} c / s_{B}$, where $\rho_{B}, s_{B}, \tau_{B}$ are the density, sound speed and Thomson optical depth at the Bondi radius, $r_{B} \sim 2 G M / s_{B}^{2}$. This should be true for black holes with masses that range from stellar values $\sim 5 \mathrm{M}_{\odot}$ to the many billion solar masses that characterize the holes that are thought to power quasars 11 国.

Four accretion states can be distinguished, although it is hard to be quantitative without a better understanding of the underlying fluid mechanics and plasma physics.

- Drought $\left(\dot{M}<<\dot{M}_{E}\right)$ The accreting gas is adiabatic. That is to say it is unable to cool and radiate away its binding energy within some transition radius $r_{\text {trans. }}$. This requires that the internal energy be carried mostly by hot ions which do not heat the electrons enough to enable them to radiate efficiently on the inflow timescale15. Instead, the surplus energy, which is transported outward by magnetic stress, is carried off in an outflow. This may account for most of the mass supply so that the hole accretion rate is very much less than the mass supply 6 .

Possible examples include low power, galactic nuclei, (including Sgr A*), AGN with powerful radio sources (including quasars) and binary X-ray sources in their low hard states [Menou, Mineshige].

- Rain $\left(\dot{M} \sim \dot{M}_{E}\right)$ The gas can radiate efficiently all the way down to the marginally stable circular orbit $r_{\mathrm{ms}}$. The flow is conservative so that the accretion rate is similar to $\dot{M} \in$. Most of the radiation will be emitted quasi-thermally by the disk, although there is likely to be considerable, nonthermal, coronal activity as well. The inner disk is radiation- and electron scattering-dominated with thickness $H \sim \dot{M} \kappa_{T} / 4 \pi c$. These disks are expected and observed to be unstable and are probably quite

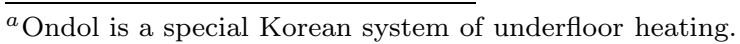


inhomogeneous. 1 The high gas density and the intense radiation field may prevent the formation of relativistic jets.

Possible examples include Seyfert galaxies, radio-quiet quasars and binary X-ray sources in their high, soft states.

- Deluge $\left(\dot{M}_{E} \lesssim \dot{M} \lesssim \dot{M}_{E}\left(c / s_{B}\right)^{2}\right)$ Radiation is trapped within the trapping radius $r_{\text {trap }} \sim \dot{M} \kappa_{T} / 4 \pi c$ within which the accreting gas flows faster than the photons can diffuse. The flow is again adiabatic and the energy that is released is carried off by a mildly relativistic, radiation-dominated wind $[$ Park $]$. The hole accretes at the roughly the Eddington rate.

Possible examples include broad absorption line quasars and Galactic superluminal sources like GRS1915+105.

- Inundation $\left(\dot{M} \gtrsim \dot{M}_{E}\left(c / s_{B}\right)^{2}\right)$ The trapping radius lies beyond $r_{B}$. Strongly super-Eddington accretion is possible because outflows from the inner disk are unable to escape.

Possible examples include massive black holes during their rapid growth phases and some GRB models.

The reason why outflows are inevitable when the flow is adiabatic is that the torque $G$ in the disk automatically transports energy radially outward at a rate $G \Omega$, where $\Omega$ is the angular velcocity 6 . There is a local heating rate $G \nabla \Omega$ which can unbind gas in a nearly Keplerian disk. I The energy liberated by each proton that (altruistically) cross the horizon drives a powerful wind from larger radii which allows many (up to $10^{5}$ ) other protons to escape.

\subsection{The Importance of Magnetic Field}

Early models of accretion disks 16 were non-specific about the nature of the viscous torque allowing it to be either fluid or hydromagnetic in character. We now know that, under almost all circumstances, the latter is correct. The magnetorotational instability 2 ensures that magnetic fields grow to dynamically important strength on the orbital timescale [Vishniac]. The nonlinear evolution of this instability is still far from clear. However, it is likely that it involves coronal heating, jet formation, and possibly emission line cloud dynamics.

${ }^{b}$ This may permit radiation to escape with a luminosity in excess of the Eddington limit. ${ }^{c}$ This is not necessarily true for a disk that extends beyond $r_{B}$ where the potential is much softer.

${ }^{d}$ It is possible that the energy is carried off hydromagnetically, in which case, there need be little mass loss. 
What is clear is that we are still quite ignorant of the true laws of MHD. The global evolution of magnetic field, as witnessed much closer to home in the terrestrial magnetosphere and the solar corona, is controlled by principles of global stability and physical processes like reconnection, conduction and equilibration, which we do not understand well enough to generalize to astrophysical environments. However, the prospects are generally quite good for improving our understanding of these matters through more detailed space physical observation, terrestrial experiments using giant pinches, lasers and particle beams as well as numerical simulation.

\subsection{Two Dimensional Adiabatic Disks}

Despite these remarks it is still instructive (and easier) to consider fluid disks. These are inevitably at least two dimensional [Lee]. A useful approximation is that the viscosity is small enough that they can be treated as being in approximate hydrostatic equilibrium. This requires specifying how the density varies along isobars which, in turn, depends upon the manner in which energy, mass and angular momentum are transported through the disk.

Two dimensional adiabatic disks are likely to become linearly unstable according to the Høiland criterion, which is a linear combination of the more familiar Schwarzschild and Rayleigh prescriptions for instability and appropriate for hot, rotating flows. There are actually two criteria. Most accretion disks are quite stable to the first of these which essentially refers to radial interchanges. It is the second criterion that is more relevant. It essentially states that fluid disks are marginally unstable to the interchange of slender rings when they are gyrentropic; that is to say when the surfaces of constant entropy and specific angular momentum coincide. (In fact this implies that the surfaces of constant Bernoulli function are also coincident.) Furthermore, the nature of the unstable modes is such that if a disk is only slightly unstable, the motions of fluid elements will transport mass, angular momentum and energy along these gyrentropes to the disk surface where they can be transferred to an outflow.

The precise properties of the outflow depend upon the detailed nature of the dissipation and momentum transfer that takes place at the disk surface and these processes are not even understood in the case of the sun. If the wind is thermally driven, then there must be an entropy jump at its base as the gas in the disk changes from being bound to unbound. However it also possible that the gas remains relatively cold and that magnetic stress create a momentum-driven wind as appears to happens in the case of the solar wind, where two million degree gas can acquire a velocity at infinity of $\sim 800 \mathrm{~km}$ 
$\mathrm{s}^{-1}$. In addition, there is the possibility of launching a cold, magnetocentrifugal wind from a near Keplerian accretion disk - a possibility that does not exist in the case of the sun. The key conclusion is that the structure of adiabatic, accretion disks depends upon the unknown physics of mass outflow.

However, even this is not the whole story. The gas that remains in the disk must flow inward on a viscous timescale and the manner in which this happens depends upon the prescription for the viscosity. Indeed, in the presence of convective motion, there is a natural quadrupolar circulation in the disk which will be established and this must be superposed upon the net flow to the disk surface and smaller radii. Just as is the case with Eddington-Sweet circulation, the flow adjusts to ensure that there is no local accumulation of mass angular momentum and energy. 目

It is possible to construct self-consistent, self-similar models that exhibit convection, inflow, outflow and circulation after specifying a functional form for the viscous torque and outflow launching mechanisnt. These models are generic and their generalfeatures are displayed by some numerical simulations of two dimensional disk17. Self-similar disks are subject to the criticism that they cannot describe the flow close to the hole, where most of the energy is released. This criticism can be met by constructing an explicit non selfsimilar, relativistic torus model around $r_{\mathrm{ms}}$. The physical assumptions that must be introduced to create such a model are necessarily arbitrary but they suffice to demonstrate that there is no difficulty of principle associated with terminating a self-similar solution at small radius. Similar concerns have been raised concerning the outer radius. Understanding this transition has turned out to be a much harder problem.

\subsection{Magnetized Disks}

We have just argued that fluid disks are gyrentropic. However, this is unlikely to be true of magnetised disks. The dynamics is clearly complex [Krolik]. For example, if it turns out that the magnetic flux preferentially settles into isorotational surfaces and distributes the angular momentum rapidly on these surfaces, then the disk structure will be barytropic. This prescription has some numerical support 18 . Whatever the correct prescription, the self-similar models described above can be modifed with only minor changes. It is the prescription for generating the outflow at the disk surface that is more important for determining the disk structure.

${ }^{e}$ If we do not admit the possibility of an outflow from the disk surface, then the global circulation will transport heat from small radii, where it is mostly liberated, to $r_{\text {trans }}$ where it must either be radiated away or continually inflate the disk so that the flow becomes unsteady. 


\section{Seungmu Dynamics}

\subsection{Ultrarelativistic Jets}

Ultrarelativistic $f^{f}$ jets are found in a variety of locales. They were first seen in extragalactic radio sources [Celotti, Sikora]. Indirect arguments, together with direct measurement of superluminal expansion show that the emitting material can move with a Lorentz factor $\Gamma \sim 10$ and be collimated into a cone with opening angle $\sim 5^{\circ}$. There is now evidence that their sources are spinning, massive black holes with their attendant accretion disks and that they are collimated within $\sim 100 \mathrm{~m}$. Most jets appear to be associated with accretion disks that radiate well below their Eddington limits and carry as much, or even more, power than is radiated by the disk. 9

The second example of ultrarelativistic jets is provided by plerions. These are supernova remnants, exempified by the Crab Nebula, with central, rapidly spinning, magnetised, neutron stars. The rotational energy of the neutron star appears to be carried off by a relativistic outflow. Recent, observations by the Chandra Observatory (now replicated in other plerions) show a pair of jet-like features, together with an equatorial disk of X-ray emission. This was surprising because it was thought that jets required an accretion disk to form. The region, that is observed directly, is clearly not moving with ultrarelativistic speed as they would then be beamed away from us. However, it is a reasonable supposition that they contain invisible, ultrarelativistic cores.

The third example is provided by GRBs which are known to be cosmologically distant and, consequently, extremely energetic. It has been inferred that the outflow is ultrarelativistic, with Lorentz factors $\Gamma \sim 300$ as $\gamma$-rays with energy in excess of $\sim 0.5 \mathrm{MeV}$ have to escape without creating pairs 14 . They are popularly associated with stellar compact objects and it was guessed that they might be beamed, in order that the burst energies not be unreasonably large. Reports of achromatic breaks in afterglow spectra from some long duration bursts, which can be formed when $\Gamma$ decreases to of order the reciprocal of the jet opening angle, support this view.

\subsection{Magnetic Rotators}

A reasonable guess is that all of these ultrarelativistic outflows (if not the more general class of cosmic jets) are due to similar physical processes. However,

${ }^{f}$ Seungmu dancers are able to beat a drum and, simultaneously, spin long tassles attached to their hats.

${ }^{g}$ It is well worth searching for the stellar counterparts of ultrarelativistic jets - Galactic blazars that are beamed towards us. 
although popular models of AGN and plerionic jets are essentially electro- or hydromagnetic, most GRB models invoke a "hot big bang" that produces a fireball with a very high entropy per baryon, just like the early universe. I would like to explore the alternative hypothesis that all three types of source are really quite similar and derive their power from the continuous extraction of rotational energy from a compact object by electromagnetic stress - a "cold, steady state" model instead of a hot, big bang! " Furthermore, I suppose that the energy remains mostly in an electromagnetic form as it is transported into the emission region.

This magnetic rotator model is best developed in the case of plerions, where it is supposed that the central spinning neutron star possesses an inclined dipole moment and that it is surrounded by a force-free magnetosphere through which currents flow and space charge is maintained [Hirotani]. The complete electrodynamical description of this magnetosphere remains an unsolved problem. However, it seems likely that somewhere beyond the light cylinder, the electromagnetic field becomes essentially axisymmetric and that variation on the scale of a wavelength dies away. There are at least three ways by which this can occur. There can be steady reconnection in the outflowing, "striped" wind. Alternatively, the waves can decay through parametric instability into higher frequency waves. These two processes are essentially dissipative. Finally, the minority of magnetic field lines that emanate from the neutron star's southern magnetic pole, and which can be traced into the northern hemisphere, may gradually be pulled by magnetic tension across the equatorial plane back into the southern hemisphere (and vice versa). This can happen non-dissipatively near the light cylinder. In summary, I will presume that only the $\mathrm{DC}$, not the $\mathrm{AC}$ component of the electromagnetic field survives.

If this simplification of the magnetic geometry actually takes place, we will be left with a relativistic wind containing a predominantly toroidal magnetic field spun off by the central body. Associated with this toroidal magnetic field will be a poloidal electrical field that is almost equal in magnitude. (Many authors have argued that this wind quickly becomes plasma-dominated and terminates in a relativistic, fluid shock front in the inner nebula. However, it is very hard to see how the DC magnetic field can be erased so quickly and, although there are moving "wisp" features, these appear to lie in the equatorial plane. There is really no evidence for a relativistic shock in tne nebula.)

There are several suggestions as to how the jets associated with extra-

${ }^{h}$ It is amusing that, if we are correct in identifying long duration GRBs with compact objects of size $\sim 10 \mathrm{~km}$, then their sources are observed to be active for a million light crossing times - an order of magnitude greater than the number of crossing times that we have observed a typical quasar! 
galactic radio sources are launched. One of the simplest is that the power for the ultrarelativistic outflow is extracted as Poynting flux from the spin of the hole by magnetic field lines that are supported by external currents flowing in the disk. It is also possible to extract energy electromagnetically from a surrounding accretion disk assuming that enough of its area is threaded by open magnetic field. However, this is most likely to produce a hydromagnetic outflow where the terminal velocity is no more than mildly relativistic although it may be responsible for collimating the much faster flow from the black hole.

When we consider electromagnetic models of GRBs, we find several advantages. The most fundamental is that the electromagnetic stress tensor, is anisotropic, in contrast to an isotropic fluid pressure tensor. This, implies that electromagnetic outflows can be naturally self-collimating. In addition, the presence of a dominant electromagnetic field implies that the effective internal sound speed is that of the fast mode which is close to the speed of light. Electromagnetic jets, in contrast to fluid jets are no more than mildly hypersonic. 月

They are few direct clues as to the prime movers of GRBs 14 . The closest model to that of AGN jets has a spinning, stellar black hole surrounding by a stellar mass torus - possibly a tidally destroyed neutron star - that confines a $\sim 10^{14} \mathrm{G}$ magnetic field [van Putten]. The emission lasts as long as the torus survives. The model that is closest to plerions involves a rapidly-spinning magnetar that has just been formed by accretion-induced collapse of a white dwarf. Alternatively, it might be possible for an accreting magnetised neutron star that is collapsing under rotational support to form a black hole or for two merging pulsars to act as magnetic rotators

The common feature of all of these putative sources is that they spin off a toroidal magnetic field and an associated, electromagnetic Poynting flux that is unburdened by baryons.

\subsection{Electromagnetic Black Holes}

The idea that the spin energy of a black hole can be extracted electromagnetically has received an observational boost from the discovery that black holes

${ }^{i}$ Contrast this with the hypernova model of GRBs 14 [Maeda, Lee] where it is supposed that a high entropy per baryon fluid is collimated by a vortex inside a star so that the pairs and $\gamma$-rays eventually transfer their energy to protons and the ratio of the momentum flux to the pressure is $>3 \Gamma^{2} \sim 3 \times 10^{5}$. This seems very unlikely to be true of a naturally occuring explosion, especially in the region where internal shocks are supposed to be operating.

${ }^{j}$ In principle, it is possible that an electromagnetic jet can be formed inside a collapsing star, though the star is not needed to provide the collimation and it seems hard to believe that plasma can be excluded from the outflow as efficiently as required. 
are commonplace on both the stellar and the massive scale (as well as, perhaps, on the intermediate scale) and that the second parameter, the spin is large so as to allow gas to orbit close to the horizon and to form strongly redshifted emission lines 22.

There are several ways through which the rotational energy associated with the spinning spacetime can be tapped electromagnetically 6 . The particular choice that I have emphasised, because I believe that it represents the dominant energy channel, is that the horizon is threaded by a large flux of open magnetic field [Park]. A continuous, electromagnetic Penrose process operates in the ergosphere of the black hole which results in Poynting flux flowing inward across the horizon and, simultaneously, propagating away from the hole to infinity. The source of the power is ultimately the reducible mass of the hole, from which the electromagnetic field in the ergosphere is extracting work.

However, not all the field lines that thread the horizon need connect with the outflow. Some low latitude field lines may connect directly to the accretion disk and provide a supplementary power source for the disk as well as a possible driving torque for exciting quasi-periodic oscillations [Dotani]. This energy channel could be important, especially if the disk is thick. However, it is unlikely to lead to an ultrarelativistic outflow. [

The process that I have just described is distinct from (though can operate simultaneously with) an alternative process, the extraction of binding energy by open field lines from the accreting gas both in the diskand in the plunging region between the inner edge of the disk and the horizon 13. The extra power that this process produces can be charged to the spin energy of the hole, which increases at a slower rate than it would do so in the absence of magnetic stress. However the intermediate working substance that effects this transformation is the inertia of the plasma not the electromagnetic field.

There are three reasons for emphasising direct extraction of energy from the hole to extraction from the infalling gas at least for a rapidly spinning hole. The first is that the event horizon has a larger effective area than the annular ring between the hole and the disk. The second is that any gas-driven outflow is likely to be contaminated with baryons and consequently is unlikely to achieve an ultrarelativistic outflow velocity required. The third is that holes probably rotate much faster than orbiting gas, except quite close to the horizon, from

\footnotetext{
$k$ The energy flow is conserved in Boyer-Lindquist coordinates and so power appears to emerge from the horizon in the Boyer-Lindquist frame. However, physical observers must orbit with respect to this coordinate system. Doppler boosting the energy flux into a frame moving with a physical observer produces an inwardly directed energy flux.

${ }^{l}$ The magnetic connection of the disk to the plunging gas seems to be a less promising source of power because thqmagnetic field lines quickly reconnect leaving the gas effectively disconnected from the disk 1 .
} 
where the extraction of energy will be quite inefficient.

\subsection{Electromagnetic Shells}

Suppose that a magnetic rotator spins off magnetic flux into the far field for a time $t_{\text {source }}$ and that this creates a relativistically expanding shell of electromagnetic field, of thickness $c t_{\text {source }}$ that drives a blast wave into the surrounding medium (Fig. 1). The blast wave is supposed to be bounded on its outside by a strong shock front that moves with Lorentz factor $\Gamma$ and, on its inside, by a contact discontinuity, separating it from the shell, that moves with Lorentz factor $\Gamma_{c}$. Suppose further, for simplicity, that the current well beyond the light cylinder flows along the axes, then along the contact discontinuity and finally returns to it source through the equatorial plane. If we ignore the poloidal component of the magnetic field, (and consequently the flux of angular momentum), then the relevant solution of the force-free equations [Park] associated with this current flow has the form

$$
\begin{aligned}
B_{\phi} & =\frac{f_{+}(t-r)+f_{-}(t+r)}{r \sin \theta} \\
E_{\theta} & =\frac{f_{+}(t-r)-f_{-}(t+r)}{r \sin \theta}
\end{aligned}
$$

The two terms in each expression are fast modes propagating outward and inward. The charge and current density vanish in the interior of the shell. We can determine the functions $f_{+}, f_{-}$by specifying the electromagnetic field at some small radius beyond the light surface and by matching to an ultrarelativistic blast wave expanding into the surrounding medium. This last requires that the outer surface of the shell move at the same speed as the inner surface of the blast wave and that the magnetic stress normal to this surface match the pressure in the blast wave.

The simplest assumption to make is that the strength of the magnetic rotator is constant, $\left(f_{+}=\right.$const $)$for a time $t_{\text {source }}$ and that the external density is constant in radius. These assumptions imply that the Lorentz factor of the blast wave's outer shock front varies with radius $R$ according to $\Gamma \propto \csc \theta R^{-1 / 2}$ 5. (The Lorentz factor of the contact discontinuity, $\Gamma_{c}$, exhibits a similar variation.) The electromagnetic velocity (the velocity of the frame in which the electric field vanishes) in the body of the shell $\vec{\beta}=\vec{E} \times \vec{B} / B^{2}$ is radial and equal in magnitude to $\left(f_{+}-f_{-}\right) /\left(f_{+}+f_{-}\right)$and the magnetic stress in a frame moving with this velocity is $\propto f_{+} f_{-} \csc ^{2} \theta$. The expansion of the blast wave is anisotropic, being faster along the poles, giving an electromagnetic power

per steradian $L_{\Omega} \propto \csc ^{2} \theta$. Note that information is propagated inward by the 
fast mode which does modify the solution and allows it to react to changes in the load including changes in its effective impedance. Note also that it takes a very long time for a wave to be reflected by the blast wave and return to the origin. This is generally true of ultrarelativistic flows and stationary solutions, which take a long time to be established can be quite misleading.

A comparison with a non-relativistic plerion like the Crab Nebula, is instructive. The magnetic bubble expands with speed of $\dot{R}<<c$ and, consequently, the pulsar must be producing magnetic flux at a rate that is roughly $(c / \dot{R})^{1 / 2}$ times too large to account for the strength of the magnetic field in the nebula. Therefore, most (95 percent in the case of the Crab Nebula) of the flux must be destroyed. On topological grounds, the natural places for this destruction to occur are on the axis and the equatorial plane. These regions are, in any case, formally unstable to pinch and tearing mode instabilities $m$ respectively 3 . The X-ray emission from the Crab Nebula, observed by Chandra21, may well be a consequence of particle acceleration associated with electrical resistance / flux destruction / generation of electromagnetic turbulence / particle acceleration tracing out the current flow in the inner part of the nebula. If we consider the flow of electromagnetic field in the nebula, we find that there must be asteady flow of magnetic flux towards the rotation axis and the equatorial plane 3 . In addition, there will be a compensatory backflow of relativistic electrons and positrons into the interior of the plerion. Of course there must be some matter in the nebula - it has been observed evaporating off filaments through its polarisation behaviour - but in this model, it has a very minor role in the dynamics.

By contrast, the contact discontinuity of an electromagnetic shell expands at a relativistic speed, and the reflected wave has a much smaller amplitude than the incident wave. Consequently, there is no necessity to destroy a lot of magnetic flux. Stated another way, there need be little resistance in the circuit. The effective load consists of the performance of work on the expanding blast wave. This is where most of the power that is generated by the central magnetic rotator ends up. (The distinction between this inertial load and a dissipative load is quite unimportant for the behavior of the black hole magnetosphere.)

The simple electromagnetic solution will only remain valid until the end of the outward-propagating, electromagnetic pulse catches up with the blast wave. This occurs at some radius $R_{\text {free }} \sim \Gamma\left(R_{\text {free }}\right)^{2} c t_{\text {source. }}$. Thereafter, the surrounding blast wave which, by now, has acquired almost all of the energy

${ }^{m}$ The contact discontinuity should be formally Kruskal-Schwarzschild stable.

${ }^{n}$ If we were to prevent this dissipation, in a thought experiment, through having the current flow along rigid, perfect conductors, then the reflected electromagnetic waves would react back on the source and shut down the power supply. 
in the explosion, will expand freely with $\Gamma \propto R^{-3 / 2}$.

This elementary model of a relativistic electromagnetic shell can be easily generalized to accommodate different assumptions about the variation of the flux supply with time and latitude and the density variation in the external medium.

\subsection{Gamma Ray Bursts}

As well as bring out some formal points, the electromagnetic solution just described provides a possible model for GRB 2012. Suppose that a magnetic rotator spins off toroidal magnetic field as it slows down and that this magnetic field fills an anisotropic, relativistically expanding shell in a uniform medium. Suppose, further, that the flux distribution near the light cylinder is consistent with the current being concentrated along the axis and in the equatorial plane, as described above. The current density is most intense on the axis and, although there is no requirement that flux be destroyed as long as the expansion is relativistic, in practice the magnetic pinch will become hydromagnetically unstable to sausage and kink modes (in the comoving frame) after expansion beyond a radius where the stabilising, poloidal field becomes insignificant 3 . These global instabilities, which should have a longitudinal wavelength comparable in size to the width of the current distribution, may sustain an electromagnetic turbulence spectrum which should ultimately be responsible for particle acceleration and the excitation of transverse gyrational motion 19. P The reason why particles are accelerated is that, when the power cascades down to short enough wavelengths, there are too few charged particles to carry the electrical current and field-parallel electric fields will develop. This is the microscopic source of the dissipation. These particles will emit $\gamma$-rays, primarily through the synchrotron process, though inverse Compton scattering may also be important. $\gamma$-rays, in excess of threshold will create fresh pairs. All of this will take place in a frame moving with the electromagnetic velocity and the emission will be strongly beamed outward. As well as create electrical resistance, the global pinch instabilities can also provide a plausible explanation for the large $\sim 1-10$ ms fluctuations in the observed $\gamma$-ray flux that are observed. p

The afterglow is formed after the blast wave becomes free of its electromagnetic driver. Now, in most afterglow models, including those involving jets, it is supposed that the expansion velocity does not vary with latitude. However,

${ }^{o}$ This turbulence may have already been seen in the measured fluctuation power spectrum [Chang].

${ }^{p}$ Attributing this variation to internal shocks tied to the source, as in the fluid model, implies that the $\gamma$-rays originate at much smaller radii than expected on the electromagnetic model. 
an electromagnetically-driven blast wave necessarily creates an anisotropic explosion and this has important consequences for observations of the afterglow, especially in the ultrarelativistic phase of expansion. If we continue to use our simple model, we find that the afterglow expansion varies most rapidly, and remains relativistic for longest, closest to the symmetry axis. As $L_{\Omega} \propto \csc ^{2} \theta$ the energy contained in each octave of $\theta$ is roughly constant This means that the most intense bursts and afterglows in a flux-limited sample will be seen pole-on and should exhibit achromatic breaks, which might be mistaken for jets. The inferred explosion energy will be roughly independent of $\theta$ and characteristic of the total energy. When the expansion becomes non-relativistic, the remnant will have a prolate shape which might be measurable. $q$

This electromagnetic model provides a solution to the puzzle of how to launch a blast wave that extends over an angular scale $>>\Gamma^{-1}$ and where the individual parts are out of causal contact. In the electromagnetic model, the energy is transferred to the blast wave by a magnetic shell that pushes (unevenly) on the surrounding gas all the way out to $R_{\text {free }}$. It also supplies an origin for the magnetic flux in the blast wave, for which the alternative origin in the bounding shock front seems very hard to explain. In the present model, magnetic field can simply be mixed into the blast wave (and the shockaccelerated relativistic electrons) at the contact discontinuity through instabilities, much like what seems to happen in regular supernova remnants.

\subsection{Some Numbers}

Let us give some illustrative orders of magnitude for a model of a long duration GRB. The electromagnetic energy flux near the pole is $L_{\Omega} \sim 10^{50} \mathrm{erg} \mathrm{s}^{-1}$ sterad $^{-1}$ and lasts for a time $t_{\text {source }} \sim 100 \mathrm{~s}$. The associated EMF in the electrical circuit $\sim 10^{22} \mathrm{~V}$. is uniform $n \sim 1 \mathrm{~cm}^{-3}$. The blast wave is driven by the electromagnetic shell with Lorentz factor $\Gamma \propto R^{-1 / 2}$ until $R \sim R_{\text {free }} \sim 10^{17} \mathrm{~cm}, \Gamma \sim \Gamma_{\text {free }} \sim 100$. Thereafter there is a freely expanding blast wave with $\Gamma \propto R^{-3 / 2}$ until the expansion becomes non-relativistic when $R \sim R_{\mathrm{NR}} \sim 3 \times 10^{18} \mathrm{~cm}$.

Most of the GRB emission (around $\sim 1 \mathrm{MeV}$ ) is produced when $R \lesssim R_{\text {free }}$ as synchrotron emission by $\sim 100 \mathrm{GeV}$ electrons in a co-moving magnetic field of strength $B \gtrsim 30 \mathrm{G}$. The comoving cooling time of these electrons is $\sim 3 \mathrm{~s}$,

${ }^{q}$ It is tempting to associate some of the barrel-shaped supernova remnants observed in our Galaxy with the remnants of electromagnetic explosions.

${ }^{r}$ A potential difference this large, made available along the contact discontinuity, provides one of the few astrophysical options for accounting for UHE cosmic rays.

${ }^{s}$ For comparison the values are $\sim 3 \times 10^{14} \mathrm{~A}, \sim 3 \times 10^{16} \mathrm{~V}$ for the Crab Nebula and $\sim 10^{18} \mathrm{~A}$, $\sim 10^{20} \mathrm{~V}$ for Cygnus A. 
a fraction $\lesssim 10^{-4}$ of the expansion timescale and so if the $\sim 100 \mathrm{GeV}$ pair energy density is maintained at a significant fraction of the equipartition energy density, then the magnetic energy can be efficiently transformed into $\gamma$-rays. The opacity to pair production for a $\gamma$-ray of energy $E$ is $\sim 0.1(E / 1 \mathrm{GeV})$. The Thomson optical depth depends upon the details of the particle acceleration but is plausibly much smaller than unity so that the observed $\gamma$-rays can freely escape without erasing the variability.

\subsection{AGN Jets}

Having briefly discussed electromagnetic models of plerions and GRBs, it remains to re-consider extragalactic jets in this context [Fletcher]. Although electromagnetic/hydromagnetic models of the energy release have been quite widely discussed for a long while, it has generally been supposed that this electromagnetic energy is transformed into a particle-dominated flow at a safe distance from the black hole and that the observed emission is from a high $\beta$ plasma.

How plausible is it that the entire radio source is an electromagnetic structure, that the observed emission trace out unstable currents rather than strong shock fronts? One of the merits of this hypothesis is that it may provide a dynamical rationalisation of the doctrine of equipartition. Pinched currents, on all scales, may continue to become unstable until their stresses are balanced by pressure. Another merit is that it provides a natural explanation for the helical structures that are often seen in VLBI maps. A third advantage is that currents can account for distributed particle acceleration in well-resolved jets, as spectral studies suggest may be required. However, if the extended lobes associated with the powerful FRII sources, like Cygnus A, are filled with unstable though fundamentally toroidal and force- free magnetic field, then this could present a quite strong signal in the Faraday rotation maps which has not really been seen. Similarly the polarisation structure of FRII radio sources does look like a shear flow and there are some features, in radio maps, like the knots in M87, which are naturally interpreted as strong shocks.

\section{Summary and Prospects}

In these impressionistic sketches, I have outlined two principles, which although not completely new, are now not commonly discussed - that most accreting black holes (excluding those where the mass supply is within an order of magnitude or so of the Eddington rate) swallow only a tiny fraction of the gas

${ }^{t}$ Of course, under force-free conditions, the currents also trace out the magnetic field in the frame in which the electric field vanishes. 
supplied at the Bondi radius and that ultrarelativistic, high energy phenomena are fundamentally electromagnetic not gas dynamical. The link between these two principles may be that carrying off the binding energy of the accreting gas in a fluid outflow, rather than radiation, is a necessary condition for extracting energy electromagnetically from a spinning black hole. At least this is the story in the case of AGN jets. This condition is also satisfied by pulsars. GRBs, which may be either accreting black holes or neutron stars, would be similar to either. An extension of this line of argument is that AGN jets may be essentially electromagnetic all the way to their hot spots.

There are many possible discriminatory observations. For the most interesting and immediate case of GRBs, it is not predicted that there will be an accompanying neutrino signal. By contrast, a gravitational wave signal is expected in some, though not all, models and would be strongly diagnostic if detected. It is unlikely, though not impossible, that GRBs will be associated with Type II supernovae. More immediate but less specific diagnostics include relating the duration and character of the GRB with the inferred observation angle of the burst - higher inclination should be associated with longer and less intense bursts. The spectrum and polarisation of the afterglow emission might also contain some clues, though the lack of a usable theory of particle acceleration ${ }^{a}$ and magnetic field amplification at ultrarelativistic shocks makes this a bit problematic. A more detailed discussion of the GRB emission, than presented here should account for the $\mathrm{MeV}$ breaks observed in $\gamma$-ray spectra.

From a more theoretical perspective, there is much to be learned about the properties of force-free electromagnetic fields and especially their stablity. The possible relationship of the GRB fluctuation power spectrum to an underlying turbulence spectrum is especially tantalising. Undoubtedly, numerical simulations will be crucial as the problem is essentially three dimensional. Force-free electromagnetism is easier to study than relativistic MHD and may well be a very good approximation in many of these sources.

Turning to plerions, the most direct, observational challenge is to see if there really is a strong, dissipating shock as expected with a fluid wind or a flow of electromagnetic energy towards the axis and the equatorial plane as predicted by the electromagnetic model and as appears to be exhibited by the Crab Nebula.

Finally, for AGN, we would like to detect and understand the mass outflows predicted above for low mass supply rates. (We already know that rapidly

${ }^{u}$ Recent, promising progress on understanding elativistic shocks predicts a power law distribution function with a logarithmic slope of 2.210 , provided that the scattering is essentially normal to the shock front. What is not yet clear is whether these scattering conditions are present. 
accreting stellar and massive holes drive dense, high speed outflows.) In addition, we need to see if the very well-observed radio jets and their lobes can be re-interpreted in terms of an unstable Z-pinch. A good place to start is through mapping the magnetic field using polarisation measurements and the internal mass density through internal depolarisation data. In addition, detailed imaging spectra from radio to X-ray energies can be used to determine where the particles are being accelerated - at shock surfaces or in volumes containing strong, unstable currents - and how they propagate away from these acceleration sites at different energies.

The conjunction of recent, impressive discoveries, upcoming observational facilities and powerful computing capability makes this a propitious time to be studying current high energy emission from black holes.

\section{Acknowledgments}

I thank the organisers of this workshop for their gracious and generous hospitality, my colleagues Mitch Begelman and Max Lyutikov for their collaboration on the above and several of the conference participants for discussion which helped to clarify some of the arguments I have presented. Support under NASA grant 5-2837 is gratefully acknowledged.

\section{References}

1. Agol, E. \& Krolik, J. H. ApJ 528, 161 (2001)

2. Balbus, S. A. \& Hawley, J. F. RMP 70, 1 (1998)

3. Begelman, M. C. ApJ 493, 291 (1998)

4. Begelman, M. C. ApJ , in press (2002)

5. Blandford, R. D. \& McKee, C. F. Phys. Fluids 19, 1130 (1976)

6. Blandford, R. D. Prog. Theor. Phys. Supp. 143, 182 (2001)

7. Blandford, R. D. Lighthouses of the Universe ed. R. Sunyaev et al Berlin:Springer-Verlag (2002)

8. Blandford, R. D. \& Begelman, M. C. MNRAS 303, L1 (1999)

9. Frank, J. H., King, A. R. \& Raine, D. J. Accretion Power in Astrophysics Cambridge: Cambridge University Press (1992)

10. Kirk, J. G., Guthmann, A. W., Gallant, Y. A. \& Achterberg, A. ApJ $\mathbf{5 4 2}, 235$ (2000)

11. Krolik, J. H. Active Galactic Nuclei Princeton: Princeton University Press (1999)

12. Lyutikov, M. \& Blackman, E. G. MNRAS 321, 177 (2001)

13. Meier, D. L., Koide, S. \& Uchida, Y. Science 291, 84 (2001)

14. Mészáros, P. ARAA, in press (2002) 
15. Narayan, R., Mahadevan, R. \& Quataert, E. The Theory of Black Hole Accretion Discs ed. M. A.. Abramowicz, G. Bjornsson \& J. E. Pringle Cambridge: Cambridge Univeristy Press p148 (1998)

16. Shakura, N. I. \& Sunyaev, R. A. Astron. Astrophys 254, 22 (1973)

17. Stone, J. M., Pringle, J. E. \& Begelman, M. C. MNRAS 310, 1002 (1999)

18. Stone, J. M. \& Pringle, J. E. MNRAS 322, 461 (2001)

19. Thompson, C. \& Blaes, O. M. Phys. Rev. D 57, 3219 (1998)

20. Usov, V. V. MNRAS 267, 1035 (1994)

21. Weisskopf, M. et alApJ 536, 81 (2000)

22. Wilms, J., Reynolds, C. S., Begelman, M. C., Reeves, R., Molendi, S., Staubert, R. \& Kendziorra, E. MNRAS 328, L27 (2001) 

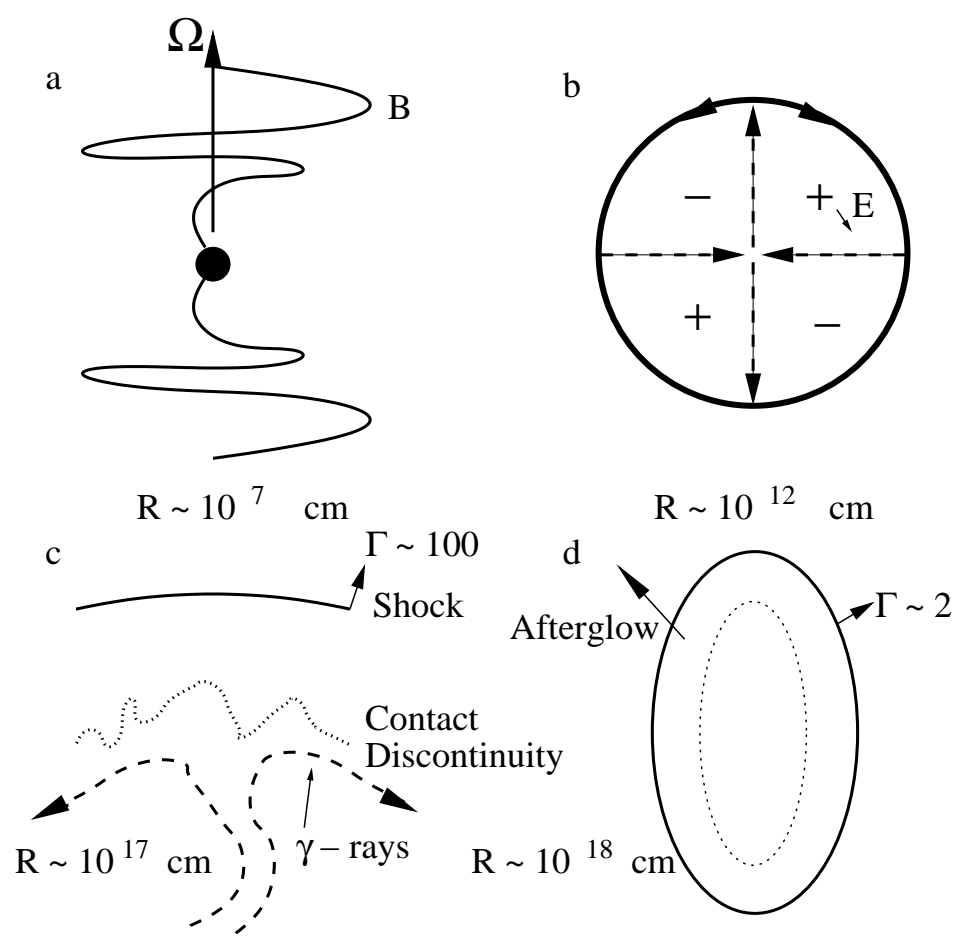

Figure 1: Four stages in the expansion of a magnetic shell with scales appropriate to a long duration GRB. a) The magnetic field $\vec{B}$ changes from poloidal to toroidal close to the outgoing light surface of the magnetic rotator at a radius $R \sim 10^{6} \mathrm{~cm}$. The alternating component of the electromagnetic field decays relative to the DC toroidal field. b) The source is active for $\sim 100 \mathrm{~s}$. By this time, it will have inflated a magnetic bubble with radius $R \sim 3 \times 10^{12} \mathrm{~cm}$, expanding with Lorentz factor $\Gamma \sim 3 \times 10^{4}$. The magnetic field is mostly toroidal, with the signs shown, while the electric field $\vec{E}$ is poloidal. The quadrupolar current flow is shown dashed. The shocked circumstellar medium is compresed into a thin shell of thickness $\sim 10^{3} \mathrm{~cm}$. c) By the time the shell has expanded to $R \sim 10^{17} \mathrm{~cm} \Gamma \sim 100$ and most of the electromagnetic pulse has caught up with the blast wave. This phase is observed a time $\sim 100 \mathrm{~s}$ after the initial explosion. The current flow is still largely quadrupolar, though it is unstable along the axis and the equator and this drives an electromagnetic turbulent cascade. which ultimately creates electrical resistance and dissipation in the form of pair production, particle acceleration and intermittent, $\gamma$-ray emission. These instablities also promote corrugation of the contact discontinuity and incorporation of the magnetic field into the shocked interstellar medium where it can mix with relativistic electrons accelerated at the bounding shock front. d) When the blast wave has expanded by a further factor ten, its speed is only mildly relativistic. Its shape will be quite prolate as the expansion is fastest along the axis. Most of the energy released by the central, spinning, magnetic rotator is now carried by the shocked interstellar medium. 